\title{
Temporal perception in visual processing as a research tool
}

\author{
Bin Zhou ${ }^{1 *}$, Ting Zhang ${ }^{2}$ and Lihua Mao ${ }^{2 *}$ \\ ${ }^{1}$ Key Laboratory of Mental Health, Institute of Psychology, Chinese Academy of Sciences, Beijing, China, ${ }^{2}$ Department of \\ Psychology, Peking University, Beijing, China
}

Accumulated evidence has shown that the subjective time in the sub-second range can be altered by different factors; some are related to stimulus features such as luminance contrast and spatial frequency, others are processes like perceptual grouping

\section{OPEN ACCESS}

Edited by:

Marc Wittmann,

Institute for Frontier Areas of Psychology and Mental Health,

Germany

Reviewed by:

Vani Pariyadath,

National Institutes of Health/National

Institute on Drug Abuse, USA

Kentaro Yamamoto,

University of Tokyo, Japan

${ }^{*}$ Correspondence:

Bin Zhou,

Key Laboratory of Mental Health, Institute of Psychology, Chinese

Academy of Sciences,

Lincui Road 16,

Beijing 100101, China

zhoub@psych.ac.cn;

Lihua Mao,

Department of Psychology, Peking

University, Yiheyuan Road 5, Beijing 100871, China maolihua@pku.edu.cn

Specialty section:

This article was submitted to

Perception Science,

a section of the journal

Frontiers in Psychology

Received: 22 February 2015 Accepted: 13 April 2015

Published: 24 April 2015

Citation:

Zhou B, Zhang T and Mao L (2015) Temporal perception in visual

processing as a research tool.

Front. Psychol. 6:521.

doi: 10.3389/fpsyg.2015.00521 and contextual modulation. These findings indicate that temporal perception uses neural signals involved in non-temporal feature processes and that perceptual organization plays an important role in shaping the experience of elapsed time. We suggest that the temporal representation of objects can be treated as a feature of objects. This new concept implies that psychological time can serve as a tool to study the principles of neural codes in the perception of objects like "reaction time (RT)." Whereas "RT" usually reflects the state of transient signals crossing decision thresholds, "apparent time" in addition reveals the dynamics of sustained signals, thus providing complementary information of what has been obtained from "RT" studies.

Keywords: subjective time, perceptual organization, research tool, neural response, object perception

\section{Introduction}

One of the extensively studied temporal topics is duration or interval timing in the sub-second range, with which a basic experience beyond instantaneity can be obtained (Fraisse, 1984). Such temporal processing is distinguished from time estimation of supra-second intervals that involves higher-order cognitive strategies (Fraisse, 1984). Whereas perceptual signal integration across tens to hundreds milliseconds is assumed to be automatic and pre-semantic (Pöppel, 1997, 2009), the feeling of how long an event lasts on this timescale appears to engage some active cognitive processes such as memory operations. Apparent time and related phenomena are often retrospectively constructed, and the context in which the temporal pattern is embedded usually exerts a modulatory effect on the time that one experiences (Eagleman, 2008; Bao et al., 2013). This retrospective and contextsensitive characteristic has also been discussed by Zhou et al. (2014a) and may have some connections with previous opinions on the phenomenological feature of subjective time (Woodrow, 1951; Gibson, 1975). Given that the spatiotemporal context often determines how stimuli are perceptually organized and perceived (Kramer and Yantis, 1997; Wagemans et al., 2012), it is important to consider the perceptual organization and its relationship to subjective time when one tries to understand the mechanisms underpinning various temporal phenomena.

\section{Sub-Second Time is Modulated by Perceptual Organization}

Traditionally, the temporal signature of an event is examined separately from the content of that event, with the assumption that time and object features are represented by independent neural substrates (e.g., Pöppel, 1997, 2009). This independence, however, may not be true, considering that 
temporal information is most likely decoded from neural signals which process object features. The spiking patterns and the signalto-noise ratio of neural activities involved in object perception thus may have an influence on the temporal decoding processes. This argument is consistent with the view that the encoding of object-identity is one of the fundamental requirements to unify different temporal phenomena (Zhou et al., 2014a). Relevant opinions are also expressed as the hypothesis that neural coding efficiency offers a basis of subjective time (Eagleman and Pariyadath, 2009). More detailed models suggest that subjective time is the output of multi-stage processes with context dependent circuits interacting with the core temporal machinery (Merchant et al., 2013). In our view, the context dependent stages include object perception processes and provide feeding signals for the computation that may function at the central stage. What is perceptually represented (i.e., the content) may alter the interaction and thus modulate how the signals are decoded.

Previous studies have accumulated evidence supporting the association of object processes and perceived time; for example, apparent duration of a visual stimulus is modulated by a preceding prime stimulus (Zhou et al., 2010). When the stimulusonset-asynchrony (SOA) is less than $40 \mathrm{~ms}$, the prime (like a disk) is likely masked by and integrated with the target (a ring with inner diameter matching the diameter of the disk), leading to expanded duration of the target. However, when the SOA increases, the prime is consciously perceived and segmented from the target, resulting in duration compression. It appears that the perceptual organization between successive stimuli distorts the apparent duration of the stimulus. A similar illusion, in a form of chronostasis, can be demonstrated in situations where a voluntary action, such as a saccadic (Yarrow et al., 2001, 2004) or a manual (Park et al., 2003; Yarrow and Rothwell, 2003) movement, triggers the onset of a stimulus whose subjective duration is ultimately expanded relative to succeeding stimuli with the same physical durations. It is assumed that the illusory duration is substantiated by a mechanism to solve the uncertainty about the sensory onset caused by movement. However, other observations show the generalization of a chronostasis-like effect in audition and vision without voluntary action (Hodinott-Hill et al., 2002; Alexander et al., 2005; Hunt et al., 2008), indicating that motor action is neither necessary nor sufficient to produce chronostasis. Instead, more general functions such as arousal level, attention, and memory are proposed to account for the induced temporal dilation. Although these notions are intriguing, they may not cover the entire picture. A likely alternative is that both action-triggered and featural changes modulate the perceptual organization of the stimulus sequence that ultimately shape the object perception and the perceived duration of the target. Observers usually compare the relative duration of the target with that of other stimuli in the train, and it is arguable that the neural system segments the stimulus train and then estimates the target duration based on the average of comparison stimuli. Indeed, some studies have shown that visual temporal averaging (Ohyama and Watanabe, 2007) and auditory rhythmic grouping (Thorpe and Trehub, 1989; Geiser and Gabrieli, 2013) alter the temporal structure of stimulus patterns and lead to relatively longer subjective intervals between compared to within perceptual groups. Our own studies with double stimuli also reveal the role of segmentation and grouping of two visual events on the apparent duration of the second event (Zhou et al., 2010, 2014b). Interestingly, the subjective duration is modulated by the dominant grouping principle (Zhou et al., 2014 b), thus manifests different patterns under various perceptual contexts.

It should be noted that the perceptual organization account does not conflict with other explanations of time distortion for brief intervals and durations. As a fundamental component of neural functions, perceptual organization profoundly interacts with other cognitive operations such as attention (Han et al., 2005; de Haan and Rorden, 2010), memory, and prior experience (Kimchi and Hadad, 2002; Zemel et al., 2002; Peterson and Berryhill, 2013). In a sense, perceptual organization under various influences sets the quality and content of our perceptual experiences (Herzog and Fahle, 2002; Wagemans et al., 2012), which may further serve as signals for the appreciation of elapsed time. On the basis of functional taxonomy (Pöppel, 1989), perceptual organization and temporal processing are logistical functions, whereas percepts represent content functions. Logistical functions usually modulate the way of contents being organized in content functions. There seems a loop that logistical functions influence content functions, which in turn carve the phenomenal representation of the former (Bao et al., 2013). Similar loop effects can be applied between logistical functions and other content functions, e.g., between the temporal organization and the content of memories. How does such loop implement itself using the "neural language"? A hypothesis advanced by Eagleman and Pariyadath (2009) may provide some clues; they argue that the consumed neural energy signals the length of the subjective duration, and neural repetition suppression is most probably underlying the time compression of repeated events. An oddball (Tse et al., 2004; Pariyadath and Eagleman, 2007, 2012; New and Scholl, 2009) or behaviorally salient stimulus (van Wassenhove et al., 2008; New and Scholl, 2009; Wittmann et al., 2010) can somehow escape the repetition suppression, thus leading to dilated apparent duration (relative to repeated stimuli). On the functional level, "oddball effects" may result from stimulus regularity and predictability (Pariyadath and Eagleman, 2007; Schindel et al., 2011) which nevertheless control the way of stimuli being perceptually organized. At what extent a stimulus can be segmented from other stimuli depends on its perceptual relation with others, on its ecological significance, or on its position in the stimulus stream. The finding that the duration of oddballs (Tse et al., 2004; Pariyadath and Eagleman, 2007, 2012), looming events (van Wassenhove et al., 2008; New and Scholl, 2009; Wittmann et al., 2010), and the first or last stimulus of a stream (Rose and Summers, 1995) is often overestimated may depend on their efficiency being separated from background events. The consequence of such process is the altered neural representation of these objects, and on the basis of the neural energy hypothesis (Eagleman and Pariyadath, 2009) their apparent durations. Similar within-sequence perceptual context and organization may also mediate the duration effect caused by speed changes even when the average speed is stable (Matthews, 2011a; Sasaki et al., 2013). Focusing on the relationship between subjective duration and perceptual organization highlights the importance of heuristic contexts and retrospective analyses on 
tracking events' time. Thus, not only immediate contexts but also task environments can modulate the apparent duration of a stimulus (Pariyadath and Eagleman, 2007; Jazayeri and Shadlen, 2010; Matthews, 2011b; Zhou et al., 2014b).

As discussed above, perceptual organization modulates subjective duration via its role on shaping the representation of objects; it is natural to ask whether object features are also able to modify the perceived lapse of time. Answering this question, the apparent duration of a stimulus has been reported to link with its size (Xuan et al., 2007; Ono and Kitazawa, 2009; Alards-Tomalin et al., 2014), intensity (Nisly and Wasserman, 1989; Xuan et al., 2007), number of elements (Xuan et al., 2007), and spatial frequency (Aaen-Stockdale et al., 2011). Interestingly, psychological time is associated with perceived qualities and quantities of objects (Ono and Kawahara, 2007; Matthews et al., 2011; Yamamoto and Miura, 2012), rather than their physical properties. For example, a central circle appears to last longer when it is surrounded by smaller than by larger circles, although it has physically identical size and duration in both conditions (Ono and Kawahara, 2007). This effect is attributed to the different apparent sizes of the central circle induced by surrounding circles, a phenomenon termed Ebbinghaus illusion. In another study (Orgs et al., 2011), viewing an implied body, but not non-body or inverted-body, motion compresses subjective time. Interestingly, the compression effect depends on the apparent length of movement paths. Taken together with the observations in oddball and repetition paradigms, these findings strongly suggest that perceptual organization operating on both spatial and temporal domains influences how the target is temporally represented relative to other objects. This view exploits factors that shape the mental time from the point of perceptual functions and provides a useful supplement to other more biological concepts and hypotheses advocated previously (Eagleman and Pariyadath, 2009; Zhou et al., 2014a). Considering its dependence on neural signals encoding objects and their identities as well as its behavioral correlation with perceived object properties, subjective time can be treated as an object feature that is indirectly computed but nevertheless can be used to tag the object identity.

\section{Subjective Time Can Serve as a Research Tool to Examine Neural Representations of Objects}

The association between the perceptual representation of objects and perceived time implies an important application of subjective time in the assessment of various mental processes. One can refer to the broad use of reaction time (RT) and its success in revealing characteristics of a variety of perceptual and cognitive operations since the beginning of experimental psychology (Pöppel, 1997; MacDonald and Meck, 2004; Posner, 2005). For a response to occur, accumulated neural signals, either detection or discrimination information, should reach a decision criterion which varies across trials but on average keeps stable for certain task sessions (Grice et al., 1982; Usher and McClelland, 2001). Usually, more strongly perceived objects lead to shorter RTs (Donner and Fagerholm, 2003; Palmer et al., 2005). Therefore, by inspecting RTs, one may obtain rich information about the neural codes of external inputs or the cognitive structures processing these inputs. In a similar vein, one can compare apparent durations of different objects under the same condition or of the same object under different conditions to study object representation and related neural processes. For example, Zhou et al. (2014b) presented observers two successive Gabor patches with varying orientation differences or spatial distances across trials. The apparent duration of the second Gabor patch is underestimated relative to its physical duration. Interestingly, the amount of duration compression is positively related to the size of orientation difference and spatial distance, in a way consistent with the orientation tuning and retinotopic mapping of early visual neurons. Similar results are also reported by Pariyadath and Eagleman (2012) when using an oddball paradigm. Thus, properties of the visual cortex can be inferred from the perceived duration of a visual stimulus without adaptation manipulation or neurophysiological recordings. The application of this concept can be found in another study (AaenStockdale et al., 2011). In a typical oddball paradigm, observers compared the relative duration of an infrequent oddball (a Gabor patch) and that of a frequent standard which could be either another Gabor patch with different spatial frequency or an auditory stimulus. The authors found an interesting result that the duration expansion or compression of the oddball depended on its spatial frequency. Relative to lower and higher spatial frequencies, a mid-range spatial frequency (ca. $2 \mathrm{c} / \mathrm{deg}$ ) consistently led to longer apparent duration that was invariant across different baseline durations. The perceived time in this example thus indicates that longer visual persistence is associated with neural responses selective to mid-range spatial frequencies in the early visual cortices. Other reports demonstrate spatially localized duration effects for drifting gratings and suggest neural sources in visual areas of striate and dorsal extrastriate cortices where neurons have specific receptive fields for moving objects (Johnston et al., 2006; Burr et al., 2007; Bruno et al., 2010). For more complex events, such as biologically relevant stimuli, studying apparent time also helps to uncover the neural processes involved in encoding these events (Wang and Jiang, 2012; Yamamoto and Miura, 2012). It has been found that merely observing the movement of another organism activates the mirror neuron system of the primate brain (Rizzolatti and Craighero, 2004). The question is whether a static image with implied motion also activates mirror neurons. Utilizing a duration judgment task, Yamamoto and Miura (2012) provide a positive answer that stationary images potentially dilate subjective time when they imply the running rather than the standing of a character. Such time effect suggests enhanced responses of mirror neurons induced by a static image with implied biological motion. In another study with pointlight walker animation (Wang and Jiang, 2012), time expansion is associated with stimuli containing biological motion components, even when observers are unaware of their biological nature. Thus, apparent duration serves as a sensitive assessment of life-relevant signals and their neural substrates. However, one has to be cautious when employing subjective time as a tool. Various factors including stimulus features, contexts, and drug administration are known to influence time perception (Meck, 1996; Eagleman, 2008) and distributed brain areas are suggested to engage in the 
coding of subjective time (Mauk and Buonomano, 2004; Merchant et al., 2013). Therefore, it is a challenge to specify neural activities which are reflected in perceived time. This limitation calls for careful design of experiments to control possible confounding factors. Consulting well-established physiological and functional properties of certain neural structures may provide help in using perceived time as a research tool.

There is another advantage to measuring apparent time in psychological and neuroscience research. Object features and identities can be processed rapidly within the first tens to hundreds milliseconds after stimulus onsets (Thorpe et al., 1996). Thus, conventional RTs primarily reflect the state of transient neural responses caused by onsets. On the other hand, duration perception needs more information that can track the lapse of event. Not only onset transients, but also sustained responses and offset transients are involved in the temporal computation. Neurophysiological studies have argued that gradual changes of neural responses, e.g., ramping activities, over frontal and parietal areas serve the neural representation of mental time (see a review by Wittmann, 2013). Although such notion needs further elaboration to accommodate current doubts and inconsistencies, it is clear that certain sustained neural responses are forwarded as time signals which could be used for phenomenal representation. To this end, apparent time serves as a tool to assess, at least partially, the sustained neural responses between onset and offset transients. However, it is important to notice that there is currently no clear delineation between the contributions of transient and sustained signals to subjective time. Considering that properties of onset transients, mainly their amplitudes, profoundly alter the representation of brief time intervals (Noguchi and Kakigi, 2006; Terao

\section{References}

Aaen-Stockdale, C., Hotchkiss, J., Heron, J., and Whitaker, D. (2011). Perceived time is spatial frequency dependent. Vision Res. 51, 1232-1238. doi: 10.1016/j.visres.2011.03.019

Alards-Tomalin, D., Leboe-McGowan, J. P., Shaw, J. D. M., and Leboe-McGowan, L. C. (2014). The effects of numerical magnitude, size, and color saturation on perceived interval duration. J. Exp. Psychol. Learn. Mem. Cogn. 40, 555-566. doi: 10.1037/a0035031

Alexander, I., Thilo, K. V., Cowey, A., and Walsh, V. (2005). Chronostasis without voluntary action. Exp. Brain Res. 161, 125-132. doi: 10.1007/s00221-004-2054-3

Bao, Y., Szymaszek, A., Wang, X., Oron, A., Pöppel, E., and Szelag, E. (2013). Temporal order perception of auditory stimuli is selectively modified by tonal and non-tonal language environments. Cognition 129, 579-585. doi: 10.1016/j.cognition.2013.08.019

Bruno, A., Ayhan, I., and Johnston, A. (2010). Retinotopic adaptation-based visual duration compression. J. Vis. 10, 30. doi: 10.1167/10.10.30

Burr, D., Tozzi, A., and Morrone, M. C. (2007). Neural mechanisms for timing visual events are spatially selective in real-world coordinates. Nat. Neurosci. 10, 423-425. doi: 10.1038/nn1874

de Haan, B., and Rorden, C. (2010). Similarity grouping and repetition blindness are both influenced by attention. Front. Hum. Neurosci. 4:20. doi: 10.3389/fnhum.2010.00020

Donner, K., and Fagerholm, P. (2003). Visual reaction time: neural conditions for the equivalence of stimulus area and contrast. Vision Res. 43, 2937-2940. doi: 10.1016/S0042-6989(03)00472-3

Eagleman, D. M. (2008). Human time perception and its illusions. Curr. Opin. Neurobiol. 18, 131-136. doi: 10.1016/j.conb.2008.06.002

Eagleman, D. M., and Pariyadath, V. (2009). Is subjective duration a signature of coding efficiency? Philos. Trans. R. Soc. Lond. B Biol. Sci. 364, 1841-1851. doi: 10.1098/rstb.2009.0026 et al., 2008), this challenge will significantly limit the application of subjective time in studies of sustained neural responses.

\section{Concluding Remarks}

Perceptual organization is an essential component of the efficient coding of the world. Here, we highlight its role in modulating the temporal representation of objects and explore its biological consequences which might underlie experiences of brief time. As a way to structure the sensory inputs, perceptual organization modifies the neural responses as well as the homeostatic conditions induced by an object. Altered biological states may change the information that is transformed into a feeling of lasting long or short (Eagleman and Pariyadath, 2009; Wittmann, 2009; Zhou et al., 2014a). In this view, perceptual organization unifies a number of time illusions on a common principle and further enables us to predict the apparent duration in a given context. The retrospective and context-dependent nature also suggests that the link between perceptual organization and subjective time is applicable in time judgment not only for online objects but also for memorized events. Using subjective time as a research tool, on the other hand, complements other behavioral measurements such as RT paradigms. With this method, it is also possible to investigate both transient and sustained neural responses encoding object identity.

\section{Acknowledgments}

The work is supported by the National Natural Science Foundation of China (Projects 31100735) and the National Basic Research Program of China (973 Program 2015CB351800).

Fraisse, P. (1984). Perception and estimation of time. Annu. Rev. Psychol. 35, 1-36. doi: 10.1146/annurev.ps.35.020184.000245

Geiser, E., and Gabrieli, J. D. E. (2013). Influence of rhythmic grouping on duration perception: a novel auditory illusion. PLOS ONE 8:e54273. doi: 10.1371/journal.pone.0054273

Gibson, J. J. (1975). "Events are perceivable but time is not," in The Study of Time II, eds J. T. Fraser and N. Lawrence (Berlin: Springer), 295-301.

Grice, G. R., Nullmeyer, R., and Spiker, V. A. (1982). Human reaction time: toward a general theory. J. Exp. Psychol. Gen. 111, 135-153. doi: 10.1037/00963445.111.1.135

Han, S., Jiang, Y., Mao, L., Humphreys, G. W., and Gu, H. (2005). Attentional modulation of perceptual grouping in human visual cortex: functional MRI studies. Hum. Brain Mapp. 25, 424-432. doi: 10.1002/hbm.20119

Herzog, M. H., and Fahle, M. (2002). Effects of grouping in contextual modulation. Nature 415, 433-436. doi: 10.1038/415433a

Hodinott-Hill, I., Thilo, K. V., Cowey, A., and Walsh, V. (2002). Auditory chronostasis: hanging on the telephone. Curr. Biol. 12, 1779-1781. doi: 10.1016/S09609822(02)01219-8

Hunt, A. R., Chapman, C. S., and Kingstone, A. (2008). Taking a long look at action and time perception. J. Exp. Psychol. Hum. Percept. Perform. 34, 125-136. doi: 10.1037/0096-1523.34.1.125

Jazayeri, M., and Shadlen, M. N. (2010). Temporal context calibrates interval timing. Nat. Neurosci. 13, 1020-1026. doi: 10.1038/nn.2590

Johnston, A., Arnold, D. H., and Nishida, S. (2006). Spatially localized distortions of event time. Curr. Biol. 16, 472-479. doi: 10.1016/j.cub.2006.01. 032

Kimchi, R., and Hadad, B.-S. (2002). Influence of past experience on perceptual grouping. Psychol. Sci. 13, 41-47. doi: 10.1111/1467-9280.00407

Kramer, P., and Yantis, S. (1997). Perceptual grouping in space and time: evidence from the Ternus display. Percept. Psychophys. 59, 87-99. doi: 10.3758/ BF03206851 
MacDonald, C. J., and Meck, W. H. (2004). Systems-level integration of interval timing and reaction time. Neurosci. Biobehav. Rev. 28, 747-769. doi: 10.1016/ j.neubiorev.2004.09.007

Matthews, W. J. (2011a). How do changes in speed affect the perception of duration? J. Exp. Psychol. Hum. Percept. Perform. 37, 1617-1627. doi: 10.1037/a0022193

Matthews, W. J. (2011b). Stimulus repetition and the perception of time: the effects of prior exposure on temporal discrimination, judgment, and production. PLoS ONE 6:e19815. doi: 10.1371/journal.pone.0019815

Matthews, W. J., Stewart, N., and Wearden, J. H. (2011). Stimulus intensity and the perception of duration. J. Exp. Psychol. Hum. Percept. Perform. 37, 303-313. doi: 10.1037/a0019961

Mauk, M. D., and Buonomano, D. V. (2004). The neural basis of temporal processing. Annu. Rev. Neurosci. 27, 307-340. doi: 10.1146/annurev.neuro.27. 070203.144247

Meck, W. H. (1996). Neuropharmacology of timing and time perception. Cogn. Brain Res. 3, 227-242. doi: 10.1016/0926-6410(96)00009-2

Merchant, H., Harrington, D. L., and Meck, W. H. (2013). Neural basis of the perception and estimation of time. Annu. Rev. Neurosci. 36, 313-336. doi: 10.1146/annurev-neuro-062012-170349

New, J. J., and Scholl, B. J. (2009). Subjective time dilation: spatially local, objectbased, or a global visual experience? J. Vis. 9, 4, 1-11. doi: 10.1167/9.2.4

Nisly, S. J., and Wasserman, G. S. (1989). Intensity dependence of perceived duration: data, theories, and neural integration. Psychol. Bull. 106, 483-496. doi: 10.1037/0033-2909.106.3.483

Noguchi, Y., and Kakigi, R. (2006). Time representations can be made from nontemporal information in the brain: an MEG study. Cereb. Cortex 16, 1797-1808. doi: $10.1093 /$ cercor/bhj117

Ohyama, J., and Watanabe, K. (2007). Unpredictable visual changes cause temporal memory averaging. Vision Res. 47, 2727-2731. doi: 10.1016/j.visres.2007.06. 020

Ono, F., and Kawahara, J.-I. (2007). The subjective size of visual stimuli affects the perceived duration of their presentation. Percept. Psychophys. 69, 952-957. doi: 10.3758/BF03193932

Ono, F., and Kitazawa, S. (2009). The effect of marker size on the perception of an empty interval. Psychon. Bull. Rev. 16, 182-189. doi: 10.3758/PBR.16.1. 182

Orgs, G., Bestmann, S., Schuur, F., and Haggard, P. (2011). From body form to biological motion: the apparent velocity of human movement biases subjective time. Psychol. Sci. 22, 712-717. doi: 10.1177/0956797611406446

Palmer, J., Huk, A. C., and Shadlen, M. N. (2005). The effect of stimulus strength on the speed and accuracy of a perceptual decision. J. Vis. 5, 376-404. doi: $10.1167 / 5.5 .1$

Pariyadath, V., and Eagleman, D. (2007). The effect of predictability on subjective duration. PLoS ONE 2:e1264. doi: 10.1371/journal.pone.0001264

Pariyadath, V., and Eagleman, D. M. (2012). Subjective duration distortions mirror neural repetition suppression. PLOS ONE 7:e49362. doi: 10.1371/journal.pone.0049362

Park, J., Schlag-Rey, M., and Schlag, J. (2003). Voluntary action expands perceived duration of its sensory consequence. Exp. Brain Res. 149, 527-529. doi: 10.1007/s00221-003-1376-x

Peterson, D. J., and Berryhill, M. E. (2013). The Gestalt principle of similarity benefits visual working memory. Psychon. Bull. Rev. 20, 1282-1289. doi: 10.3758/s13423-013-0460-x

Pöppel, E. (1989). "Taxonomy of the subjective: an evolutionary perspective," in Neuropsychology of Visual Perception, ed J. W. Brown (Hillsdale NJ: Lawrence Erlbaum Associates), 219-232.

Pöppel, E. (1997). A hierarchical model of temporal perception. Trends Cogn. Sci. 1, 56-61. doi: 10.1016/S1364-6613(97)01008-5

Pöppel, E. (2009). Pre-semantically defined temporal windows for cognitive processing. Philos. Trans. R. Soc. Lond. B Biol. Sci. 364, 1887-1896. doi: 10.1098/ rstb.2009.0015

Posner, M. I. (2005). Timing the brain: mental chronometry as a tool in neuroscience. PLoS Biol. 3:e51. doi: 10.1371/journal.pbio.0030051

Rizzolatti, G., and Craighero, L. (2004). The mirror-neuron system. Annu. Rev. Neurosci. 27, 169-192. doi: 10.1146/annurev.neuro.27.070203.144230

Rose, D., and Summers, J. (1995). Duration illusions in a train of visual stimuli. Perception 24, 1177-1187. doi: 10.1068/p241177

Sasaki, K., Yamamoto, K., and Miura, K. (2013). The difference in speed sequence influences perceived duration. Perception 42, 198-207. doi: 10.1068/p7241
Schindel, R., Rowlands, J., and Arnold, D. H. (2011). The oddball effect: perceived duration and predictive coding. J. Vis. 11, 17. doi: 10.1167/11.2.17

Terao, M., Watanabe, J., Yagi, A., and Nishida, S. (2008). Reduction of stimulus visibility compresses apparent time intervals. Nat. Neurosci. 11, 541-542. doi: 10.1038/nn.2111

Thorpe, L. A., and Trehub, S. E. (1989). Duration illusion and auditory grouping in infancy. Dev. Psychol. 25, 122-127. doi: 10.1037/0012-1649.25.1.122

Thorpe, S., Fize, D., and Marlot, C. (1996). Speed of processing in the human visual system. Nature 381, 520-522. doi: 10.1038/381520a0

Tse, P. U., Intriligator, J., Rivest, J., and Cavanagh, P. (2004). Attention and the subjective expansion of time. Percept. Psychophys. 66, 1171-1189. doi: 10.3758/BF03196844

Usher, M., and McClelland, J. L. (2001). The time course of perceptual choice: the leaky, competing accumulator model. Psychol. Rev. 108, 550-592. doi: 10.1037/0033-295X.108.3.550

van Wassenhove, V., Buonomano, D. V., Shimojo, S., and Shams, L. (2008). Distortions of subjective time perception within and across senses. PLoS ONE 3:e1437. doi: 10.1371/journal.pone.0001437

Wagemans, J., Elder, J. H., Kubovy, M., Palmer, S. E., Peterson, M. A., Singh, M., et al. (2012). A century of Gestalt psychology in visual perception I. Perceptual grouping and figure-ground organization. Psychol. Bull. 138, 1172-1217. doi: $10.1037 / \mathrm{a} 0029333$

Wang, L., and Jiang, Y. (2012). Life motion signals lengthen perceived temporal duration. Proc. Natl. Acad. Sci. U.S.A. 109, E673-E677. doi: 10.1073/pnas. 1115515109

Wittmann, M. (2009). The inner experience of time. Philos. Trans. R. Soc. Lond. B Biol. Sci. 364, 1955-1967. doi: 10.1098/rstb.2009.0003

Wittmann, M. (2013). The inner sense of time: how the brain creates a representation of duration. Nat. Rev. Neurosci. 14, 217-223. doi: 10.1038/nrn3452

Wittmann, M., van Wassenhove, V., Craig, A. D., and Paulus, M. P. (2010). The neural substrates of subjective time dilation. Front. Hum. Neurosci. 4:2. doi: 10.3389/neuro.09.002.2010

Woodrow, H. (1951). “Time perception," in Handbook of Experimental Psychology, ed. S. S. Stevens (Oxford: Wiley), 1224-1236.

Xuan, B., Zhang, D., He, S., and Chen, X. (2007). Larger stimuli are judged to last longer. J. Vis. 7, 1-5. doi: 10.1167/7.10.2

Yamamoto, K., and Miura, K. (2012). Time dilation caused by static images with implied motion. Exp. Brain Res. 223, 311-319. doi: 10.1007/s00221-012-3259-5

Yarrow, K., Haggard, P., Heal, R., Brown, P., and Rothwell, J. C. (2001). Illusory perceptions of space and time preserve cross-saccadic perceptual continuity. Nature 414, 302-305. doi: 10.1038/35104551

Yarrow, K., Johnson, H., Haggard, P., and Rothwell, J. C. (2004). Consistent chronostasis effects across saccade categories imply a subcortical efferent trigger. J. Cogn. Neurosci. 16, 839-847. doi: 10.1162/089892904970780

Yarrow, K., and Rothwell, J. C. (2003). Manual chronostasis: tactile perception precedes physical contact. Curr. Biol. 13, 1134-1139. doi: 10.1016/S09609822(03)00413-5

Zemel, R. S., Behrmann, M., Mozer, M. C., and Bavelier, D. (2002). Experiencedependent perceptual grouping and object-based attention. J. Exp. Psychol. Hum. Percept. Perform. 28, 202-217. doi: 10.1037/0096-1523.28.1.202

Zhou, B., Pöppel, E., and Bao, Y. (2014a). In the jungle of time: the concept of identity as a way out. Front. Psychol. 5:844. doi: 10.3389/fpsyg.2014.00844

Zhou, B., Qin, J., Mao, L., Han, S., and Pöppel, E. (2010). Modulations of temporal perception by consciously and unconsciously perceived stimuli. Perception 39, 900-908. doi: 10.1068/p6662

Zhou, B., Yang, S., Mao, L., and Han, S. (2014b). Visual feature processing in the early visual cortex affects duration perception. J. Exp. Psychol. Gen. 143, 1893-1902. doi: $10.1037 / \mathrm{a} 0037294$

Conflict of Interest Statement: The authors declare that the research was conducted in the absence of any commercial or financial relationships that could be construed as a potential conflict of interest.

Copyright (C) 2015 Zhou, Zhang and Mao. This is an open-access article distributed under the terms of the Creative Commons Attribution License (CC BY). The use, distribution or reproduction in other forums is permitted, provided the original author(s) or licensor are credited and that the original publication in this journal is cited, in accordance with accepted academic practice. No use, distribution or reproduction is permitted which does not comply with these terms. 